EESTI NSV TEADUSTE AKADEEMIA TOIMETISED. $\mathrm{X}$ KÖIDE

FOCSIKALIS-MATEMAATILISTE JA TEHNILISTE TEADUSTE SEERIA, 1961, NR. 1

ИЗВЕСТИЯ АКАДЕМИИ НАУК ЭСТОНСКОИ ССР. ТОМ Х СЕРИЯ ФИЗИКО-МАТЕМАТИЧЕСКИХ И ТЕХНИЧЕСКИХ НАУК. 1961, № 1

\title{
О СХОДИМОСТИ ПРИБЛИЖЕННЫХ МЕТОДОВ ИНТЕРПОЛЯЦИОННОГО ТИПА ДЛЯ ОБЫКНОВЕННЫХ ДИФФЕРЕНЦИАЛЬНЫХ УРАВНЕНИЙ
}

\section{И. ПЕТЕРСЕН,}

\section{кандидат физико-математических наук}

1. Задачей, рассматриваемой ниже, является решение дифференциального уравнения

$$
L x \equiv x^{(2 m)}(t)-\lambda\left[p_{1}(t) x^{(2 m-1)}(t)+\ldots+p_{2 m}(t)\right]=y(t)
$$

при граничных условиях

$$
\begin{gathered}
x(-1)=x^{\prime}(-1)=\ldots=x^{(m-1)}(-1)=0, \\
x(1)=x^{\prime}(1)=\ldots=x^{(m-1)}(1)=0 .
\end{gathered}
$$

Приближенное решение этой задачи будем искать в виде полинома, удовлетворяющего граничным условиям

$$
\tilde{x}=\left(1-t^{2}\right)^{m} \sum_{i=1}^{N} c_{i} t^{i-1}
$$

в котором $c_{1}, c_{2}, \ldots, c_{N}$ определяются условием, что уравнение (1.1) удовлетворяется в каком-то смысле на некоторой заданной системе узлов $t_{1}, t_{2}, \ldots, t_{n}$ интервала $[-1 ; 1]$. Такие приближенные методы естественно назвать и н те рп оля ци он ны ми.

2. Э. Б. Карпиловской [1,2] установлены условия сходимости ме тода совпадения, т. е. интерполяционного метода в частном случае $N=n$, когда $n$ коэффициентов $c_{i}$ определяются $n$ уравнениями

$$
[L \tilde{x}-y]_{t=t_{j}}=0 \quad(j=1,2, \ldots, n) .
$$

К. Б. Биценко и Р. Граммель [3] предложили метод подобл а с те й, при котором $N=n-1$ и условие (2.1) заменяется уравнениями

$$
\int_{t_{j}}^{t_{j+1}}(L \tilde{x}-y) d t=0 \quad(j=1,2, \ldots, n-1)
$$

Предложим еще три метода интерполяционного типа:

А. Берется $N=2 n-1$ и коэффициенты $c_{i}$ определяются уравнениями (2.1) и (2.2). 
В. Берется $N=2 n$ и коэффициенты $c_{i}$ определяются уравнениями (2.1) и

$$
\left[\frac{d}{d t}(L \tilde{x}-y)\right]_{t=t_{j}}^{-}=0 \quad(\mathrm{j}=1,2, \ldots, n) .
$$

С. Берется $N=2 n$ и коэффициенты $c_{i}$ определяются * уравнениями (2.1) и

$$
\left[\frac{d^{2 m+1} \tilde{x}}{d t^{2 m+1}}\right]_{t=t_{j}}=0 \quad(j=1,2, \ldots, n) .
$$

Для простоты ограничимся при рассмотрении этих методов интерполяционными узлами Чебышева.

3. Следуя идеям Л. В. Канторовича, рассмотрим задачу (1.1) (1.2) как функциональное уравнение

$$
L x \equiv G x-\lambda T x=y,
$$

где

$$
G x=\frac{d^{2 m} x}{d t^{2 m}}, \quad T x=\sum_{i=1}^{2 m} p_{i} \frac{d^{2 m-i} x}{d t^{2 m-i}}
$$

операторы $G$ и $T$ отображают нормированное пространство $X$ в нормированное пространство $Y$, причем $X$ состоит из функций, удовлетворяющих граничным условиям (1.2), и

$$
\|x\|_{X}=\|G x\|_{Y}
$$

Уравнения для определения коэффициентов $c_{i}$ в (1.3) означают при каждом методе «приближенное» уравнение

$$
G \tilde{x}-\lambda \Phi T \tilde{x}=\Phi \tilde{y}
$$

где $\tilde{x}$ и $\tilde{y}$ - элементы некоторых полных подпространств $\tilde{X}=\tilde{X}(n) \subset X$ и $\tilde{Y}=\tilde{Y}(n) \subset Y$ соответственно, причем $G(\tilde{X)}=\tilde{Y}$, и $\Phi=\Phi(n)$ есть некоторый линейный оператор проектирования $Y$ на $\tilde{Y}$.

Если операторы $T$ и $L^{-1}$ линейны и для всякого $x \in X$ найдется такое $\tilde{y} \in \tilde{Y}$, что

и такое $\tilde{y} \epsilon \tilde{Y}$, что

$$
\|T x-\tilde{y}\|_{Y} \leqslant \mu_{1}\|x\|_{X}
$$

причем

$$
\|y-\tilde{y}\| \leqslant \mu_{2}
$$

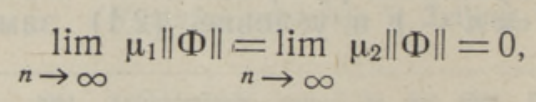

то, по Канторовичу [1], уравнение (3.4) имеет для всех достаточно больших $n$ единственное решение $\tilde{x}^{*}$, сходящееся при $n \rightarrow \infty$ к решению $x^{*}$ уравнения (3.1) со скоростью

- Метод С является методом совпадения, но не с такнм видом $\tilde{x}$, как рассмотренный в ${ }^{1,2}$, $]$. 


$$
\left\|x^{*}-\tilde{x}^{*}\right\|_{X}=O(\varepsilon\|\Phi\|),
$$

где $\varepsilon$ такое, что найдется $\tilde{x} \in \tilde{X}$, при котором

$$
\left\|x^{*}-\tilde{x}\right\|_{X} \leqslant \varepsilon .
$$

4. Введем условия:

I. $\lambda$ не является собственным числом задачи $(1.1)-(1.2)$.

II. $p_{1}(t)=0 . *$

III. Коэффициенты $p_{2}(t), \ldots, p_{2 m}(t)$ и свободный член $y(t)$ на $[-1 ; 1] r$ раз непрерывно дифференцируемы и их производные порядка $r$ удовлетворяют условию Липшица с показателем $\alpha$.

IV. Существует такая постоянная $A$, что на $(-1 ; 1)$

$$
\frac{\left|p_{2}(t)\right|}{\sqrt{1-t^{2}}} \leqslant A
$$

V. Узлы $t_{1}, t_{2}, \ldots, t_{n}$ - нули полинома Чебышева $T_{n}(t)$.

Те о рем а 1. Если выполнены условия $\mathrm{I}-\mathrm{V}$, то при $r \geqslant 1, \alpha>0$ уравнения (2.2) для определения n-го приближения ** задачи (1.1) (1.2) методом подобластей однозначно разрешимы для всех достаточно больщих $n$. Получаемые приближения и их производные, до порядка $2 m-1$ включительно, сходятся равномерно на $[-1 ; 1]$, а производные порядка $2 \mathrm{~m}$ сходятся равномерно на всяком интервале $[a ; b] \subset(-1 ; 1) \kappa$ решению задачи $(1.1)-(1.2)$ и к его соответствуюцим производным. Или, более подробно,

$$
\begin{aligned}
& \max _{t}\left|\frac{d^{k} x^{*}}{d t^{k}}-\frac{d^{k} \tilde{x}^{*}}{d t^{k}}\right|=O\left(\frac{\ln n}{n^{r+\alpha-1}}\right) \quad(k=0,1, \ldots, 2 m-1), \\
& \max _{t}\left(\left|\frac{d^{2 m} x^{*}}{d t^{2 m}}-\frac{d^{2 m} \tilde{x}^{*}}{d t^{2 m}}\right| \sqrt{1-t^{2}}\right)=O\left(\frac{\ln n}{n^{r+\alpha-1}}\right),
\end{aligned}
$$

где $x^{*}$ точное решение задачи $(1.1)-(1.2)$, а $\tilde{x}^{*}$ n-ое приближение по методу подобластей.

Док а з а те ль ств о. Для применения общей теории, кратко сформулированной в 3 , возьмем в качестве пространства $Y$ пространство $Y_{1}$ всех непрерывных на $[-1 ; 1]$ функций с нормой

$$
\|y\|_{Y_{1}}=\max _{t}\left|y(t) \sqrt{1-t^{2}}\right|
$$

* Если $p_{1}(t) \neq 0$, но $2 m-1$ раз непрерывно дифференцируема, то путем подходящей подстановки всегда можно добиться выполнения условия II.

** Под порядком приближения понимаем число узлов. 
Соответствующим пространством $X$ является тогда пространство $X_{1}$ на $[-1 ; 1] 2 m$ раз непрерывно дифференцируемых и удовлетворяющих условиям (1.2) функций с нормой

$$
\|x\|_{X_{1}}=\max _{t}\left|x^{(2 m)}(t) \sqrt{1-t^{2}}\right| .
$$

Пусть $g(s, t)$ функция Грина оператора $G$. Еслй $y=G x$, то

$$
\begin{array}{r}
x(s)=\int_{-1}^{+1} g(s, t) y(t) d t, \quad x^{(k)}(s)=\int_{-1}^{+1} \frac{\partial^{k}}{\partial s^{k}} g(s, t) y(t) d t, \\
(k=0,1, \ldots, 2 m-1)
\end{array}
$$

и на основании ограниченности частых производных $\frac{\partial^{k}}{\partial s^{k}} g(s, t)$ до порядка $2 m-1$ имеем

$$
\begin{aligned}
& \max _{s}\left|x^{(k)}(s)\right| \leqslant \max _{s} \int_{-1}^{+1} \frac{\mid \frac{\partial^{k}}{\partial s^{k}} g(s, t)}{\sqrt{1-t^{2}}}\left|y(t) \sqrt{1-t^{2}}\right| d t \leqslant \\
& \leqslant \max _{s} \int_{-1}^{+1} \frac{\mid \frac{\partial^{k}}{\partial s^{k}} g(s, t)}{\sqrt{1-t^{2}}} d t \cdot \max _{t}\left|y(t) \sqrt{1-t^{2}}\right|
\end{aligned}
$$

Следовательно,

$$
\max _{t}\left|x^{(k)}(t)\right| \leqslant A_{k}\|x\|_{X_{1}} \quad(k=0,1, \ldots, 2 m-1) .
$$

Из (4.5), I и III (при $r>0$ ) легко заключить ограниченность операторов $T$ и $L^{-1}$ относительно норм (4.3) и (4.4).

Подпространство $\tilde{X}_{1}$ состоит из всех полиномов вида

$$
\left(1-t^{2}\right)^{m} \sum_{i=1}^{n-1} c_{i} t^{i-1}
$$

а $\tilde{Y}_{1}$ - из всех полиномов степени не выше $n-2$. Оператор Ф относит к каждой непрерывной функции $y \in Y_{1}$ такой полином $\mathcal{L}[y ; t]$ степени не выше $n-2$, что

$$
\int_{i_{j}}^{t_{j+1}} \mathscr{L}[y ; t] d t=\int_{t_{j}}^{t_{j+1}} y(t) d t \quad(j=1,2, \ldots, n-1) .
$$

Имеем

$$
\mathscr{L}[y ; t]=\frac{d}{d t} L[z ; t]
$$

где $L[z ; t]-$ интерполяционный полином Лагранжа функции

$$
z(t)=\int_{0}^{t} y(t) d t
$$


по узлам $t_{1}, t_{2}, \ldots, t_{n}$, так как

$$
\int_{t_{j}}^{t_{j+1}} \frac{d}{d t} L[z ; t] d t=L\left[z ; t_{j+1}\right]-L\left[z ; t_{j}\right]=z\left(t_{j+1}\right)-z\left(t_{j}\right)=\int_{t_{j}}^{t_{j+1}} y(t) d t .
$$

Однозначность и аддитивность оператора Ф очевидны. Оценим его норму. Прежде всего по (4.8) имеем

$$
\max _{t}|z(t)|=\max _{t}\left\|_{0}^{t} \frac{1}{\sqrt{1-t^{2}}} y(t) \sqrt{1-t^{2}} d t \mid \leqslant \frac{\pi}{2}\right\| y \|_{Y_{1}}
$$

Дальше известно [4], что для интерполяционного полинома Лагранжа в случае узлов Чебышева имеет место оценка

$$
\max _{t}|L[z ; t]| \leqslant \max _{t}|z(t)| O(\ln n) .
$$

Наконец, по теореме Бернштейна,

$\|\Phi y\|_{Y_{1}}=\max \left|\mathcal{L}[y ; t] \sqrt{1-t^{2}}\right| \leqslant(n-1) \max |L[z ; t]| \leqslant \frac{\pi}{2}(n-1) O(\ln n)\|y\|_{Y_{1}}$.

Следовательно,

$$
\|\Phi\|=O(n \ln n) .
$$

Пусть теперь выполнены условия II, III, IV с $r \geqslant 1, \alpha>0$ и при $i=2,3, \ldots, 2 m$

$$
\left|p_{i}(t)\right| \leqslant L_{i},\left|p_{i}^{\prime}(t)\right| \leqslant M_{i},\left|p_{i}^{\prime}\left(t^{\prime}\right)-p_{i}^{\prime}\left(t^{\prime \prime}\right)\right| \leqslant N_{i}\left|t^{\prime}-t^{\prime \prime}\right|^{\alpha} .
$$

Если $T x=z, z(t)=\sum_{i=2}^{2 m} p_{i}(t) x^{(2 m-i)}(t), \quad$ то из $(4.11),(4.5)$ и (4.1) следует

$$
\begin{aligned}
& \left|z^{\prime}\left(t^{\prime}\right)-z^{\prime}\left(t^{\prime \prime}\right)\right| \leqslant \sum_{i=2}^{2 m}\left[\left|p_{i}^{\prime}\left(t^{\prime}\right)\right|\left|x^{(2 m-i)}\left(t^{\prime}\right)-x^{(2 m-i)}\left(t^{\prime \prime}\right)\right|+\right. \\
& \left.+\left|x^{(2 m-i)}\left(t^{\prime \prime}\right)\right|\left|p_{i}^{\prime}\left(t^{\prime}\right)-p_{i}^{\prime}\left(t^{\prime \prime}\right)\right|\right]+\sum_{i=2}^{2 m}\left|p_{i}\left(t^{\prime}\right) x^{(2 m-i+1)}\left(t^{\prime}\right)-p_{i}\left(t^{\prime \prime}\right) x^{(2 m-i+1)}\left(t^{\prime \prime}\right)\right| \leqslant \\
& \leqslant \sum_{i=2}^{2 m}\left(M_{i}\left|x^{(2 m-i+1)}\left(\tau_{i}^{\prime}\right)\right|\left|t^{\prime}-t^{\prime \prime}\right|+N_{i}\left|x^{(2 m-i)}\left(t^{\prime \prime}\right)\right|\left|t^{\prime}-t^{\prime \prime}\right|^{\alpha}\right)+ \\
& +\sum_{i=2}^{2 m}\left|p_{i}^{\prime}\left(\tau_{i i}^{\prime \prime}\right) x^{(2 m-i+1)}\left(\tau_{i}^{\prime \prime}\right)+p_{i}\left(\tau_{i}^{\prime \prime}\right) x^{(2 m-i+2)}\left(\tau_{i}^{\prime \prime}\right)\right|\left|t^{\prime}-t^{\prime \prime}\right| \leqslant \\
& \leqslant\left[\sum_{i=2}^{2 m}\left(M_{i} A_{2 m-i+1}\left|t^{\prime}-t^{\prime \prime}\right|{ }^{1-\alpha}+N_{i} A_{2 m-i}\right)\right]\|x\|_{X_{1}}\left|t^{\prime}-t^{\prime \prime}\right|^{\alpha}+ \\
& +\left[\sum_{i=3}^{2 m}\left(M_{i} A_{2 m-i+1}+L_{i} A_{2 m-i+2}\right)\right]\|x\|_{X_{1}}\left|t^{\prime}-t^{\prime \prime}\right|+\left[M_{2} A_{2 m-1}\|x\|_{X_{1}}+\right. \\
& \left.+\frac{\left|p_{2}\left(\tau_{2}^{\prime \prime}\right)\right|}{\sqrt{1-\tau_{2}^{\prime \prime 2}}}\left|x^{(2 m)}\left(\tau_{2}^{\prime \prime}\right)\right| \sqrt{1-\tau_{2}^{\prime \prime 2}}\right]\left|t^{\prime}-t^{\prime \prime}\right| \leqslant C\|x\|_{X_{1}}\left|t^{\prime}-t^{\prime \prime}\right|{ }^{\alpha} .
\end{aligned}
$$


Таким образом, $\frac{d}{d t} T x$ удовлетворяет условию Липшица с показателем $\alpha$ и с постоянной $C\|x\|_{X_{1}^{*}}$. По теореме Джексона тогда существует такой полином $\tilde{y} \in \tilde{Y}_{1}$, что

$$
\|T x-\tilde{y}\|_{Y_{1}} \leqslant \max _{t}|T x-\tilde{y}|=O\left(\frac{1}{n^{1+\alpha}}\right)\|x\|_{X_{1}} .
$$

Из III при $r \geqslant 1, \alpha>0$ следует также, что и для свободного члена $y(t)$ существует $\tilde{y} \in \tilde{Y}_{1}$ такой, что

$$
\|y-\tilde{y}\|_{Y_{1}} \leqslant \max _{t}|y-\tilde{y}|=O\left(\frac{1}{n^{1+\alpha}}\right)
$$

Оценки $(4.12),(4.13)$ и (4.10) показывают, что условия $(3.5),(3.6)$ и (3.7) выполнены.

Для доказательства утверждения (4.2) отметим, что из III следует $(2 m+r)$-кратная непрерывная дифференцируемость решения $x^{*}$ нашей задачи и что его производная порядка $2 m+r$ удовлетворяет условию Липшица с показателем $\alpha$. По теореме Джексона, тогда существует такое $\tilde{x} \in \tilde{X}_{1}$, что

$$
\left\|x^{*}-\tilde{x}\right\|_{X_{1}} \leqslant \max _{t}\left|\frac{d^{2 m} x^{*}}{d t^{2 m}}-\frac{d^{2 m} \tilde{x}}{d t^{2 m}}\right|=O\left(\frac{1}{n^{r+\alpha}}\right) .
$$

Ввиду этого (4.2) следует теперь из (3.8), (4.5) и (4.10).

5. Для метода А в качестве пространств $X$ и $Y$ возьмем те же $X_{1}$ и $Y_{1}$, что и в 4. Подпространство $\tilde{X}_{1}$ в этом случае состоит из всех полиномов вида $\left(1-t^{2}\right)^{m} \sum_{i=1}^{2 n-1} c_{i} t^{i-1}$, а $\tilde{Y}_{1}-$ из всех полиномов степени не выше $2 n-2$. Оператор Ф относит теперь к непрерывной функции $y \in Y_{1}$ полином $\mathscr{X}[y ; t]$ степени не выше $2 n-2$, однозначно определенный условиями

$$
\begin{array}{cc}
\mathscr{H}\left[y ; t_{j}\right] & =y\left(t_{j}\right) \quad(j=1,2, \ldots, n), \\
\int_{t_{j}}^{t_{j}+1} \mathscr{E}[y ; t] d t & =\int_{t_{j}}^{t_{j}+1} y(t) d t \quad(j=1,2, \ldots, n-1) .
\end{array}
$$

Если опять $z(t)=\int_{0}^{t} y(t) d t$, а $H[z ; t]$ - ее интерполяционный полином Әрмита по узлам $t_{1}, t_{2}, \ldots, t_{n}$, т. е.

$$
H[z ; t]=\sum_{i=1}^{n} z\left(t_{i}\right) A_{i}(t)+z^{\prime}\left(t_{i}\right) B_{i}(t)
$$

где $A_{i}(t)$ и $B_{i}(t)$ - основные функции эрмитовой интерполяции, то

$$
\mathscr{X}[y ; t]=\frac{d}{d t} H[z ; t],
$$

так как

$$
\left.\frac{d}{d t} H[z ; t]\right|_{t=t_{j}}=z^{\prime}\left(t_{j}\right)=y\left(t_{j}\right)
$$


и

$$
\int_{t_{j}}^{t_{j+1}} \frac{d}{d t} H[z ; t] d t=H\left[z ; t_{j+1}\right]-H\left[z ; t_{j}\right]=z\left(t_{j+1}\right)-z\left(t_{j}\right)=\int_{t_{j}}^{t_{j+1}} y(t) d t .
$$

Для оценки $\|\Phi\|$ воспользуемся (5.2), теоремой Бернштейна, (4.9), неравенством

$$
\left|z^{\prime}(t)\right|=|y(t)| \leqslant \frac{\max _{t}\left|y(t) \sqrt{1-t^{2}}\right|}{\sqrt{1-t^{2}}}=\frac{\|y\|_{Y_{1}}}{\sqrt{1-t^{2}}}
$$

и тем, что при узлах Чебышева $\left[^{4}\right]$

$$
\sum_{i=1}^{n}\left|A_{i}(t)\right|=1, \quad \max _{t} \sum_{i=1}^{n} \frac{\left|B_{i}(t)\right|}{\sqrt{1-t_{i}^{2}}}=O\left(\frac{\ln n}{n}\right) .
$$

Имеем

$$
\begin{aligned}
& \qquad\|\Phi y\|_{Y_{1}}=\max _{t}\left|\mathcal{H}[y ; t] \sqrt{1-t^{2}}\right| \leqslant(2 n-1) \underset{t}{\max _{t}|H[z ; t]| \leqslant} \\
& \leqslant(2 n-1)\left[\frac{\pi}{2}\|y\|_{Y_{1}} \max _{t} \sum_{i=1}^{n}\left|A_{i}(t)\right|+\|y\|_{Y_{1}} \max _{t \cdot} \sum_{i=1}^{n} \frac{\left|B_{i}(t)\right|}{\sqrt{1-t^{2}}}\right]=O(n)\|y\|_{Y_{1}}, \\
& \text { так что }
\end{aligned}
$$

$$
\|\Phi\|=O(n)
$$

На основании 4 , в частности $(4.12)-(4.14)$, и $(5.4)$ имеет место

Т еорем а 2. Если выполнены условия I-V, то при $r \geqslant 1, \alpha>0$ уравнения (2.1) - (2.2) для определения n-го приближения задачи (1.1) - (1.2) методом А однозначно разрешимы для всех достаточно больших $n$ и эти приближения $\tilde{x}^{*}$ сходятся $\kappa$ точному решению $x^{*}$ со скоростью

$$
\begin{gathered}
\max _{t}\left|\frac{d^{k} x^{*}}{d t^{k}}-\frac{d^{k} \tilde{x}^{*}}{d t^{k}}\right|=O\left(\frac{1}{n^{r+\alpha-1}}\right) \quad(k=0,1, \ldots, \quad 2 m-1), \\
\max _{t}\left(\left|\frac{d^{2 m} x^{*}}{d t^{2 m}}-\frac{d^{2 m} \tilde{x}^{*}}{d t^{2 m}}\right| \sqrt{1-t^{2}}\right)=O\left(\frac{1}{n^{r+\alpha-1}}\right) .
\end{gathered}
$$

6. Приступим к изучению сходимости метода В. Пространство $Y_{2}$ определяем как пространство всех один раз непрерывно дифференцируемых на $[-1 ; 1]$ функций с нормой

$$
\|y\|_{Y_{2}}=\max _{t}|y(t)|+\max _{t}\left|y^{\prime}(t) \sqrt{1-t^{2}}\right| .
$$

Соответствующее пространство $X_{2}$ состоит тогда из всех $2 m+1$ раз непрерывно дифференцируемых на $[-1 ; 1]$ функций, удовлетворяющих условиям (1.2). При этом по (3.3)

$$
\|x\|_{X_{2}}=\max _{t}\left|x^{(2 m)}(t)\right|+\max _{t}\left|x^{(2 m+1)}(-t) \sqrt{1-t^{2}}\right| .
$$

При исследовании метода совпадения для задачи (1.1) - (1.2) в [1] установлено, что

$$
\max _{t}\left|x^{(k)}(t)\right| \leqslant A_{k} \max \left|x^{(2 m)}(t)\right| \quad(k=0,1, \ldots, 2 m) .
$$


Согласно (6.2) и (6.3) имеем

$$
\begin{gathered}
\max _{t}\left|x^{(k)}(t)\right| \leqslant A_{k}\|x\|_{X_{2}} \quad(k=0,1, \ldots, 2 m), \\
\max _{t}\left|x^{(2 m+1)}(t) \sqrt{1-t^{2}}\right| \leqslant A_{2 m+1}\|x\|_{X_{2}} .
\end{gathered}
$$

Если условия I и III выполнены с $r \geqslant 1$, то при помощи (6.4) не трудно установить ограниченность операторов $T$ и $L^{-1}$.

Соответствующий методу В оператор Ф относит к дифференцируемой функции $y \in Y_{2}$ ее интерполяционный полином Эрмита $H[y ; t]$ по узлам $t_{1}, t_{2}, \ldots, t_{n}$. Следовательно. на основании теоремы Бернштейна и $(5.3)$

$$
\begin{gathered}
\|\Phi y\|_{Y_{2}}=\max _{t}|H[y ; t]|+\max _{t}\left|H^{\prime}[y ; t] \sqrt{1-t^{2}}\right| \leqslant \\
\leqslant(1+2 n-1)\left|\max _{t}\right| H[y ; t] \mid \leqslant 2 n\left[\max _{t}|y(t)| \cdot \max _{t} \sum_{i=1}^{n}\left|A_{i}(t)\right|+\right. \\
\left.+\max _{t}\left|y^{\prime}(t) \sqrt{1-t^{2}}\right| \cdot \max _{t} \sum_{i=1}^{n} \frac{\left|B_{i}(t)\right|}{\sqrt{1-t_{i}^{2}}}\right]=O(n)\|y\|_{Y_{2}}
\end{gathered}
$$

и поэтому

$$
\|\Phi\|=O(n) .
$$

Если выполнены условия II, III и IV с $r \geqslant 2, \alpha>0$, то можно аналогично 4 показать, что $\frac{d^{2}}{d t^{2}} T x$ удовлетворяет условию Липшица с показателем $\alpha$ и постоянной $D\|x\|_{X_{2}}$, причем $D$ не зависит от $x$. Тогда существует такой полином $\tilde{y} \in \tilde{Y}_{2}$ степени не выше $2 n-1$, что одновременно

$$
\max _{t}|T x-\tilde{y}|=O\left(\frac{1}{n^{1+\alpha}}\right)\|x\|_{X_{2}} \text { и } \max _{t}\left|\frac{d}{d t}(T x-\tilde{y})\right|=O\left(\frac{1}{n^{1+\alpha}}\right)\|x\|_{X_{z}}
$$

и, следовательно,

$$
\|T x-\tilde{y}\|_{Y_{2}}=O\left(\frac{1}{n^{1+\alpha}}\right)\|x\|_{X_{2}}
$$

В то же время существует и такой $\tilde{y}_{\epsilon} \tilde{Y}_{2}$, что

$$
\|y-\tilde{y}\|_{Y_{2}}=O\left(\frac{1}{n^{1+\alpha}}\right) \text {. }
$$

Из (6.5), (6.6) и (6.7) видно, что (3.7) выполнено.

На основании III имеем в (3.9) в рассматриваемом случае

$$
\varepsilon=O\left(\frac{1}{n^{r+q-1}}\right)
$$

Таким образом доказана

Т еорем а 3. Если выполнены условия I-V, то при $r \geqslant 2, \alpha>0$ уравнения $(2.1)-(2.3)$ для определения $n$-го приближения задачи (1.1) - (1.2) методом В однозначно разрешимы для всех достаточно больших $n$ и эти приближения $\tilde{x}^{*}$ сходятся $\kappa$ точному решеншю $x^{*}$ со скоростью 


$$
\begin{aligned}
& \max _{t}\left|\frac{d^{k} x^{*}}{d t^{k}}-\frac{d^{k} \tilde{x}^{*}}{d t^{k}}\right|=O\left(\frac{1}{n^{r+\alpha-2}}\right) \quad(k=0,1, \ldots, 2 m), \\
& \max _{t}\left(\left|\frac{d^{2 m+1} x^{*}}{d t^{2 m+1}}-\frac{d^{2 m+1} \tilde{x}^{*}}{d t^{2 m+1}}\right| \sqrt{1-t^{2}}\right)=O\left(\frac{1}{n^{r+\alpha-2}}\right) .
\end{aligned}
$$

7. Те орем а 4. Если $\lambda$ не является собственным числом задачи $(1.1)-(1.2), p_{1}(t), \ldots, p_{2 m}(t)$ и у $(t)$ на $[-1 ; 1]$ непрерывны $и$ удовлетворяют условию Липшица $c$ положительным показателем $\alpha$, то метод С при узлах Чебышева применим к приближенному решению этой задачи при всех достаточно больших п. Приближенные решения при этом сходятся к точному со скоростью

$$
\max _{t}\left|\frac{d^{k} x^{*}}{d t^{k}}-\frac{d^{k} \tilde{x}^{*}}{d t^{k}}\right|=O\left(n^{-\frac{\alpha}{2}}\right) \quad(k=0,1, \ldots, 2 m) .
$$

Доказательств о. Пусть $Y_{3}-$ пространство непрерывных на $[-1 ; 1]$ функций с обычной нормой

$$
\|y\|_{Y_{\mathrm{a}}}=\max _{t}|y(t)| .
$$

Тогда в $X_{3}$ согласованная норма определена равенством

$$
\|x\|_{x_{3}}=\max _{t}\left|x^{(2 m)}(t)\right| .
$$

В связи с методом совпадения показано ['], что операторы $T$ и $L^{-1}$ (если $\lambda$ не собственное число задачи) ограничены относительно норм (7.2) и (7.3).

Подпространство $\tilde{X}_{3}$, соответствующее методу С, состоит из всех полиномов вида $\left(1-t^{2}\right)^{m} \sum_{i=1}^{2 n} c_{i} t^{i-1}$, удовлетворяющих условию

а подпространство $\tilde{Y}_{3}-$ из всех полиномов степени не выше $2 n-1$, первые производные которых имеют нулями $t_{1}, t_{2}, \ldots, t_{n}$.

Оператор Ф относит к любой функции $y(t)$ из $Y_{3}$ ее интерполяционный полином Фейера $F[y ; t]$, определенный условиями

$$
F\left[y ; t_{j}\right]=y\left(t_{j}\right),\left.\frac{d}{d t} F[y ; t]\right|_{t=t_{j}}=0 \quad(j=1,2, \ldots, n) .
$$

Как известно,

$$
F[y ; t]=\sum_{i=1}^{n} y\left(t_{i}\right) A_{i}(t)
$$

По (5.3) для узлов Чебышева имеем

$$
\|\Phi\|=O(1) \text {. }
$$

Если функция $y(t)$ на $[-1 ; 1]$ непрерывна и удовлетворяет условию Липшица с показателем $\alpha>0$ и постоянной $K$, то для узлов Чебышева имеет место оценка [5]

$$
\max _{t}|y(t)-F[y ; t]| \leqslant 5 \cdot 2^{\alpha} K n^{-\frac{\alpha}{2}} .
$$

Из (7.5) следует, что условие (3.6) выполнено с $\mu_{2}=O\left(n^{-\frac{\alpha}{2}}\right)$. 
В рассматриваемом теоремой случае $T_{x}$ удовлетворяет условию Липшица с показателем $\alpha$ и постоянной $E\|x\|$, где $E$ не зависит от $x$. Поэтому и (3.5) выполнено, причем $\mu_{1}=O\left(n^{-\frac{\alpha}{2}}\right)$. Из-за (7.4) условие (3.7) выполнено. Наконец, для (3.9) имеем $\varepsilon=O\left(n^{-\frac{\alpha}{2}}\right)$, так что (7.1) следует из (3.8) и (6.3). Тем самым теорема доказана.

\title{
ЛИТ Е Р АТ У РА
}

1. Л. В. Канторович, Г. П. Акилов, Функциональный анализ в нормированных пространствах, Физматгиз, 1959.

2. Э. Б. К а рпилов ск ая, Успехи матем. наук, т. VIII, вып. 3 (55), 1953, $111-118$.

3. К. Б. Бицен ко, Р. Г р а м ме ль, Техническая динамика, т. І, Гостехиздат, 1950.

4. И. П. Н а тан сон, Конструктивная теория функций, Гостехиздат, 1949.

5. O. Shisha, C. Sternin, M. Fekete, Riveon lemat., 8, 1954, 59-64.

\author{
Институт кибернетики \\ Академии наук Эстонской ССР
}

Поступила в редакцию 15. VII 1960

\section{INTERPOLATSIOONITUOPI LIGIKAUDSETE MEETODITE KOONDUVUSEST HARILIKE DIFERENTSIAALVÕRRANDITE PUHUL}

\section{Petersen,}

füüsikalis-matemaatiliste teaduste kandidaat

Resümee

Artiklis vaadeldakse rajaülesande $(1.1)$ - (1.2) ligikaudse lahendamise meetodeid, milles lahend otsitakse polünoomina (1.3) ja kordajad $c_{i}$ määrab tingimus, et võrrand (1.1) oleks teataval viisil rahuldatud antud sōlmedes $t_{1}, t_{2}, \ldots, t_{n}$. Osapiirkondade meetodi [ [3] puhul on ligikaudne lahend määratud tingimustega (2.2), meetodi A puhul tingimustega (2.1) ja $(2.2)$, meetodi $B$ puhul tingimustega (2.1) ja (2.3) ning meetodi $C$ puhul tingimustega (2.1) ja (2.4). Kasutades üldist ligikaudsete meetodite teooriat ['], antakse nende nelja meetodi koonduvustingimused ja koonduvuskiiruse hinnangud Tšebõševi sõlmede juhul.

15. VII 1960

\section{UBER DIE KONVERGENZ DER ANNÄHERUNGSMETHODEN VOM INTERPOLATIONSTYPUS FUR GEWÖHNLICHE DIFFERENTIALGLEICHUNGEN}

\section{Petersen}

\section{Zusammenfassung}

In der Abhandlung werden einige angenäherte Lösungsmethoden der Grenzwertaufgabe (1.1) - (1.2) betrachtet, die die Lösung in Form eines Polynoms (1.3) suchen, wobei die Koeffizienten $c_{i}$ durch die Bedingung bestimmt sind, dass die Gleichung (1.1) in gewissem Sinne in gegebenen Knotenpunkten $t_{1}, t_{2}, \ldots, t_{n}$ erfüllt ist. Bei der Teilgebietsmethode $\left[^{3}\right]$ ist die angenäherte Lösung durch die Bedingungen (2.2), bei der Methode A durch (2.1) und (2.2), bei der Methode B durch (2.1) und (2.3), bei der Methode C durch (2.1) und (2.4) bestimmt. Auf Grund der allgemeinen Theorie der Annäherungsmethoden [ $\left.{ }^{1}\right]$ werden die Konvergenzbedingungen dieser vier Methoden festgestellt und die Abschätzung ihrer Konvergenzgeschwindigkeit im Falle der Tschebyschevschen Knotenpunkte durchgefürt. 